\title{
Nebular phase observations of the Type-lb supernova iPTF13bvn favour a binary progenitor ${ }^{\star}$
}

\author{
H. Kuncarayakti ${ }^{1,2}$, K. Maeda ${ }^{3,4}$, M. C. Bersten ${ }^{5,4}$, G. Folatelli5 ${ }^{5,4}$, N. Morrell ${ }^{6}$, E. Y. Hsiao ${ }^{6}$, S. González-Gaitán ${ }^{1,2}$ \\ J. P. Anderson ${ }^{7}$, M. Hamuy ${ }^{2,1}$, T. de Jaeger ${ }^{1,2}$, C. P. Gutiérrez ${ }^{1,2,7}$, and K. S. Kawabata ${ }^{8,9}$ \\ ${ }^{1}$ Millennium Institute of Astrophysics, Casilla 36-D, Santiago, Chile \\ e-mail: hanin@das.uchile.cl \\ 2 Departamento de Astronomía, Universidad de Chile, Casilla 36-D, Santiago, Chile \\ 3 Department of Astronomy, Kyoto University, Kitashirakawa-Oiwake-cho, Sakyo-ku, 606-8502 Kyoto, Japan \\ ${ }^{4}$ Kavli Institute for the Physics and Mathematics of the Universe (WPI), The University of Tokyo, Kashiwa, 277-8583 Chiba, Japan \\ 5 Instituto de Astrofísica de La Plata (IALP), CCT-CONICET-UNLP, Paseo del Bosque S/N, B1900FWA La Plata, Argentina \\ ${ }^{6}$ Las Campanas Observatory, Carnegie Observatories, Casilla 601, La Serena, Chile \\ 7 European Southern Observatory, Alonso de Córdova 3107, Vitacura, Casilla 763 0355, Santiago, Chile \\ ${ }^{8}$ Hiroshima Astrophysical Science Center, Hiroshima University, Higashi-Hiroshima, 739-8526 Hiroshima, Japan \\ 9 Core of Research for the Energetic Universe, Hiroshima University, Higashi-Hiroshima, 739-8526 Hiroshima, Japan
}

Received 31 December 2014 / Accepted 7 April 2015

\section{ABSTRACT}

\begin{abstract}
Aims. We present and analyse late-time observations of the Type-Ib supernova with possible pre-supernova progenitor detection, iPTF13bvn, which were done $\sim 300$ days after the explosion. We discuss them in the context of constraints on the supernova's progenitor. Previous studies have proposed two possible natures for the progenitor of the supernova, i.e. a massive Wolf-Rayet star or a lower-mass star in a close binary system.

Methods. Our observations show that the supernova has entered the nebular phase, with the spectrum dominated by $\mathrm{Mg} \mathrm{I}] \lambda \lambda 4571$, [O I] $\lambda \lambda 6300,6364$, and [Ca II] $\lambda \lambda 7291,7324$ emission lines. We measured the emission line fluxes to estimate the core oxygen mass and compared the $[\mathrm{O} \mathrm{I}] /[\mathrm{Ca} \mathrm{II}]$ line ratio with other supernovae.

Results. The core oxygen mass of the supernova progenitor was estimated to be $\lesssim 0.7 M_{\odot}$, which implies initial progenitor mass that does not exceed $\sim 15-17 M_{\odot}$. Since the derived mass is too low for a single star to become a Wolf-Rayet star, this result lends more support to the binary nature of the progenitor star of iPTF13bvn. The comparison of $[\mathrm{O} \mathrm{I}] /[\mathrm{Ca}$ II] line ratio with other supernovae also shows that iPTF13bvn appears to be in close association with the lower mass progenitors of stripped-envelope and Type-II supernovae.
\end{abstract}

Key words. supernovae: general - supernovae: individual: iPTF13bvn - stars: massive

\section{Introduction}

Supernovae (SNe) Types Ib and Ic, which do not show hydrogen lines in their spectra, are thought to be produced by the core collapse of massive stars that have lost their outer hydrogen envelope. Hydrogen-deficient Wolf-Rayet (WR) stars have been proposed as the possible progenitors for these SNe (Maeder \& Lequeux 1982; Ensman \& Woosley 1988). However, thus far there has been no strong confirmation of a WR star as a SN Ib/c progenitor. As summarized by Eldridge et al. (2013), examinations of Hubble Space Telescope (HST) pre-SN archival images of $\mathrm{SNe} \mathrm{Ib} / \mathrm{c}$ in nearby galaxies have not succeeded in detecting the progenitor stars. Yoon et al. (2012) argue that Type-Ib/c SN progenitors are faint in optical bands during the pre-SN stage,

\footnotetext{
* Based on observations obtained at the Southern Astrophysical Research (SOAR) telescope, which is a joint project of the Ministerio da Ciência, Tecnologia, e Inovação (MCTI) da República Federativa do Brasil, the US National Optical Astronomy Observatory (NOAO), the University of North Carolina at Chapel Hill (UNC), and Michigan State University (MSU); Chilean Telescope Time Allocation Committee proposal CN2014A-91.
}

thus direct detections have not been providing strong constraints on the progenitor luminosity and mass.

Cao et al. (2013) report the discovery and early evolution of iPTF13bvn, a Type-Ib SN discovered by the intermediate Palomar Transient Factory (iPTF) survey (Rau et al. 2009; Law et al. 2009) in the host galaxy NGC 5806. It was found in the HST archives that the SN position coincides with a blue source, within $2 \sigma$ (80 mas, $\approx 8.7 \mathrm{pc})$ positional uncertainty of the SN. The photometry of the object was shown to be consistent with a WR star, which was suggested as the progenitor of iPTF13bvn. Groh et al. (2013) propose that their model of WR star with initial mass of 31-35 $M_{\odot}$ could reproduce the observed photometry of the object, the candidate progenitor star of iPTF13bvn. If the disappearance of the candidate progenitor star is confirmed by future observations, this would be the first detection of a progenitor of a Type-Ib SN.

An alternative scenario is proposed by Bersten et al. (2014), in which the progenitor star was modelled to be in a close binary system. Their hydrodynamic model shows that the primary star in this system evolved, lost its hydrogen layer due to binary interaction, and exploded as a $\approx 3.5 M_{\odot}$ pre-SN star, corresponding 
Table 1. Observing log.

\begin{tabular}{lrcc}
\hline \hline Object & Exp. time (s) & Airmass & Seeing $\left({ }^{\prime \prime}\right)$ \\
\hline iPTF13bvn spec. & 5100 & $1.4-1.2$ & $1.2-0.9$ \\
HR5501 spec. & 1 & 1.2 & 0.9 \\
iPTF13bvn $B V R_{\mathrm{c}}$ & 300 & $1.2-1.3$ & $0.9-1.2$ \\
iPTF13bvn $U$ & 1800 & 1.3 & 1.2 \\
PG1528 $B V R_{\mathrm{c}}$ & $1-3$ & 1.4 & 1.3 \\
PG1528 $U$ & 30 & 1.4 & 1.3 \\
PG1525 $B V R_{\mathrm{c}}$ & $2-5$ & 1.3 & 1.2 \\
PG1525 $U$ & 30 & 1.3 & 1.2 \\
\hline
\end{tabular}

Notes. HR5501 is a spectrophotometric standard star; PG1525 and PG1528 are Landolt photometric standard fields.

to the progenitor initial mass of $20 M_{\odot}$. This model successfully reproduced the observed light curve, absence of hydrogen, and photometry of the pre-SN candidate progenitor star. The notion that the progenitor star of iPTF13bvn could not have been a massive WR star, but instead a lower mass star in a binary system, has also been suggested by Fremling et al. (2014) and Srivastav et al. (2014). Eldridge et al. (2015) argue that the photometry of Cao et al. (2013) underestimated the brightness of the candidate progenitor object by up to $0.7 \mathrm{mag}$, which led them to the conclusion that the observed spectral energy distribution does not match a typical single WR star, but is instead more consistent with their binary models.

In this paper we present the optical photometry and spectroscopy of iPTF13bvn obtained during its nebular phase, at 306 days after the explosion date, from which we derive an estimate of the initial mass of the SN progenitor star.

\section{Observations and data reduction}

The observations of iPTF13bvn were carried out using the $4.1 \mathrm{~m}$ Southern Astrophysical Research (SOAR) telescope stationed at Cerro Pachón, Chile at an altitude of $2700 \mathrm{~m}$ above sea level, starting in the night of 18 April 2014 (Chilean local time) in visitor mode. This observation date corresponds to $\sim 306$ days after the assumed explosion time of JD $=2456459.24 \pm 0.9$ (Bersten et al. 2014), or +290 days past the time of maximum light.

We used the Goodman High Throughput Spectrograph (Clemens et al. 2004) for both photometry and spectroscopy. The Goodman HTS employs a Fairchild 4k $\times 4 \mathrm{k}$ CCD with a physical scale of $0.15^{\prime \prime} /$ pixel. The unvignetted field of view size of the instrument is around 7.2' in diameter. We used the $2 \times 2$ binning mode for both photometry and spectroscopy. During the night, the sky was clear but not photometric with variable seeing. Table 1 shows the observation log for the night. The observation of iPTF13bvn was started with spectroscopy, followed by photometry. Spectrophotometric standard star and photometric standard fields (Landolt 1992) were also observed during the night.

We used the 300 lines/mm grating with GG385 blocking filter and 1.03" slit mask, positioned at the parallactic angle, for our spectroscopic observation of iPTF13bvn. With this instrument configuration, the dispersion for $2 \times 2$ pixel binning is $2.6 \AA /$ pixel with wavelength coverage $3600-8800 \AA$. We took a $1500 \mathrm{~s}$ exposure and $2 \times 1800 \mathrm{~s}$ exposures of iPTF13bvn, resulting in a total of $5100 \mathrm{~s}$ exposure time for the object. We used the $3^{\prime \prime}$ slit for the spectrophotometric standard star. An internal $\mathrm{HgAr}$ arc lamp was used for wavelength calibration, and an internal quartz lamp was used for spectral flat calibration. From the arc spectra we measured the effective full width at half maximum (FWHM) resolution of the spectra to be $\approx 7.6 \AA$ around the wavelength $7000 \AA$. The raw data was reduced using IRAF $^{1}$ in a standard slit-spectroscopy reduction manner, with the TWODSPEC and ONEDSPEC packages. Wavelength and flux calibrations were also performed.

Photometry was carried out in $U B V R \mathrm{c}$ bands. Figure 1 shows the position of the $\mathrm{SN}$ within its host galaxy. The $\mathrm{SN}$ is clearly detected in the $R \mathrm{c}$ band, as well as in $B$ and $V$, although not as clearly. In the $U$ band, the SN is not detected within $2 \sigma$ of the background noise. The integration time was $300 \mathrm{~s}$ for the $B V R \mathrm{c}$ filters, and $1800 \mathrm{~s}$ for the $U$ filter. Point-spread function (PSF) photometry was performed on the $B V R \mathrm{c}$ images and aperture photometry on the $U$-band image using an aperture value equal to the seeing size, after standard image reduction procedures with IRAF. PSF photometry was performed using the DAOPHOT package (Stetson 1987) and aperture photometry using APPHOT.

\section{Results and discussions}

\subsection{Spectroscopy}

Our observation was conducted 306 days after the SN explosion. At this age, the $\mathrm{SN}$ is expected to have already entered the nebular phase, which is confirmed by the observed spectrum $^{2}$ (Fig. 2). The continuum of the spectrum is very weak with strong emission lines of [O I] $\lambda \lambda 6300,6364$ and [Ca II] $\lambda \lambda 7291$, 7324 dominating. In addition to the [O I] and [Ca II] lines, the spectrum also exhibits a noticeable $\mathrm{Mg}$ I] $\lambda 4571$ semi-forbidden intercombination line. These spectral features are commonly shown by stripped-envelope core-collapse SNe in the nebular stage (see Fig. 2). Nebular spectra of seven other SNe (Modjaz et al. 2008, 2014; Maeda et al. 2007b, 2008) are shown alongside iPTF13bvn for comparison in Fig. 2.

The nebular spectrum of iPTF13bvn looks remarkably similar to the type-IIb SN 2008ax at +307 days post-maximum, despite the noticeably weaker signal-to-noise ratio. SNe IIb are thought to be closely related to $\mathrm{SNe} \mathrm{Ib}$ - both subclasses show prominent helium lines, but the hydrogen lines present in type$\mathrm{IIb} \mathrm{SNe}$ are observed only during the early phase. Thus, it is possible that many SNe IIb might have been misidentified as a Type-Ib. Indeed it has been suggested that the IIb or Ib classification may be time-dependent (Milisavljevic et al. 2013). In the case of iPTF13bvn, hydrogen was not detected in spectra as early as +2.3 days after the explosion, hence the Ib classification (Cao et al. 2013); however, see the discussions about possible $\mathrm{H} \alpha$ emission in Sect. 3.1.3.

\subsubsection{The $[\mathrm{O} I]$ line profile}

The [O I] $\lambda \lambda 6300,6364$ emission line of iPTF13bvn evidently does not show a single-peak, Gaussian-like profile. It exhibits a rather boxy, flat-topped profile that to some extent is suggestive of a double-peaked emission. In Fig. 3 we compare the line profile of the [O I] $\lambda \lambda 6300,6364$ emission in iPTF13bvn to that of SN 2008ax at the same phase. The spectrum of SN 2008ax is the same as the one presented in Fig. 2. Both spectra show [O I] FWHM of $\sim 5000 \mathrm{~km} \mathrm{~s}^{-1}$. While the [Ca II] line of iPTF13bvn

\footnotetext{
1 IRAF is distributed by the National Optical Astronomy Observatory, which is operated by the Association of Universities for Research in Astronomy (AURA) under cooperative agreement with the National Science Foundation.

2 Available at the WISeREP database,

http://wiserep.weizmann.ac.il/
} 
H. Kuncarayakti et al.: Nebular phase observations of the Type-Ib supernova iPTF13bvn favour a binary progenitor

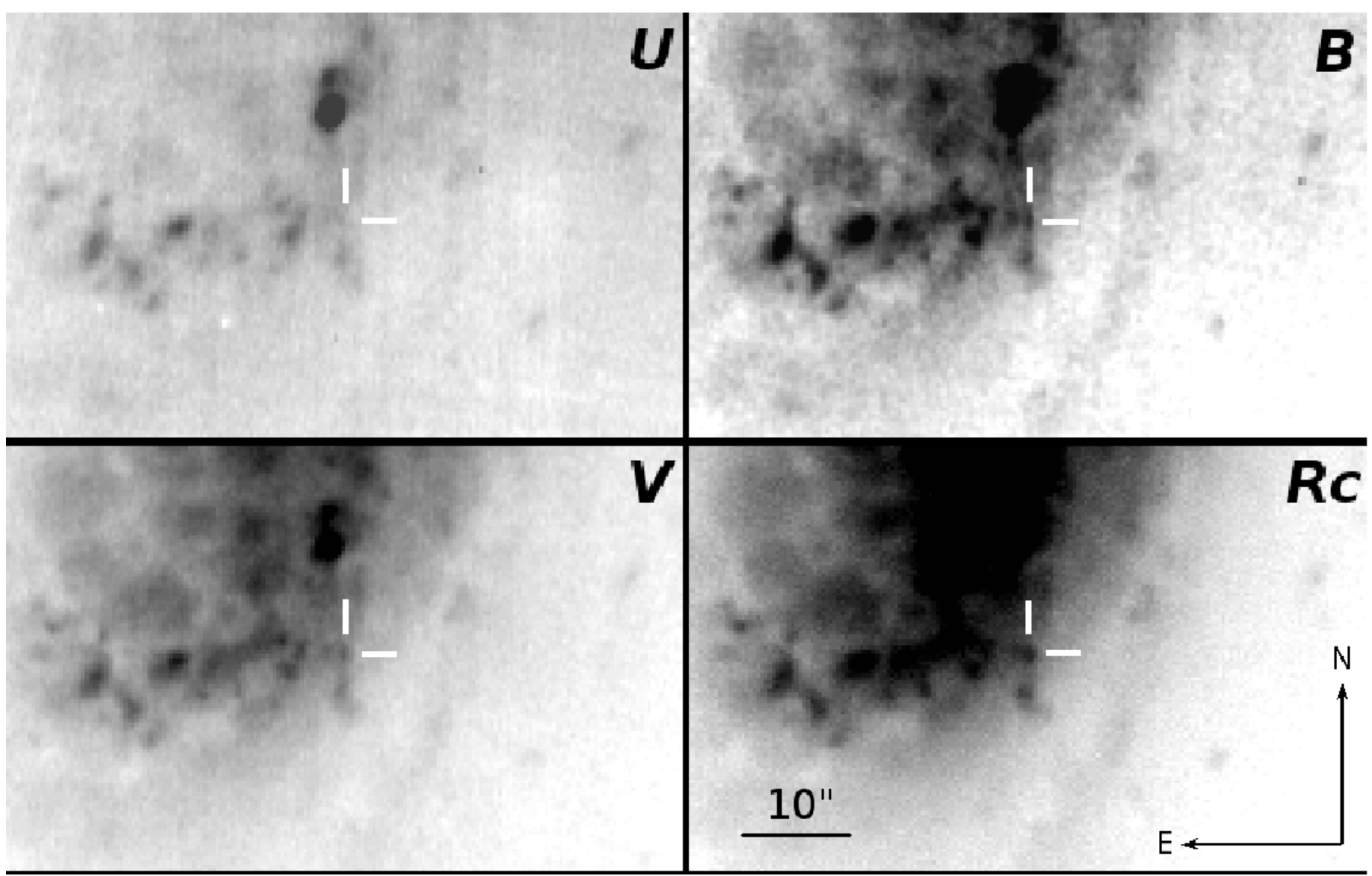

Fig. 1. Sections of $U B V R$ c images of iPTF13bvn, with the SN indicated.

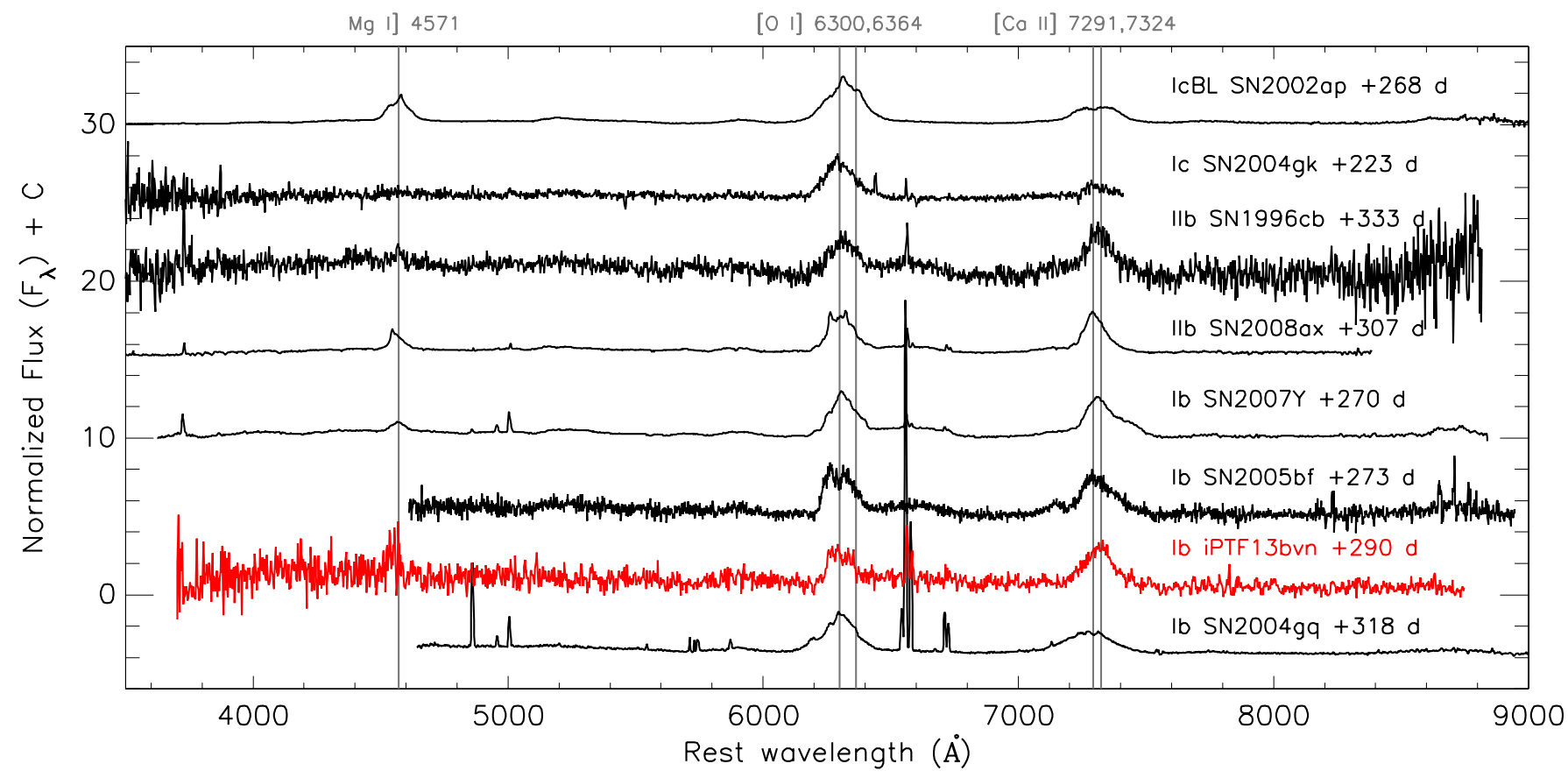

Fig. 2. Spectrum of iPTF13bvn at 306 days past explosion (+290 days after maximum light), compared to several stripped-envelope core-collapse SNe at similar ages from Modjaz et al. (2008, 2014), Maeda et al. (2007b, SN 2005bf), and Maeda et al. (2008, SN 2004gq). The phase of the spectra in days are with respect to the time of maximum light. The prominent emission lines that come from the SNe are indicated with vertical grey lines. The spectra are normalized to the peak of the [O I] line of iPTF13bvn and shifted in flux for presentation purposes. The narrow emission lines originate from the underlying H II regions. 


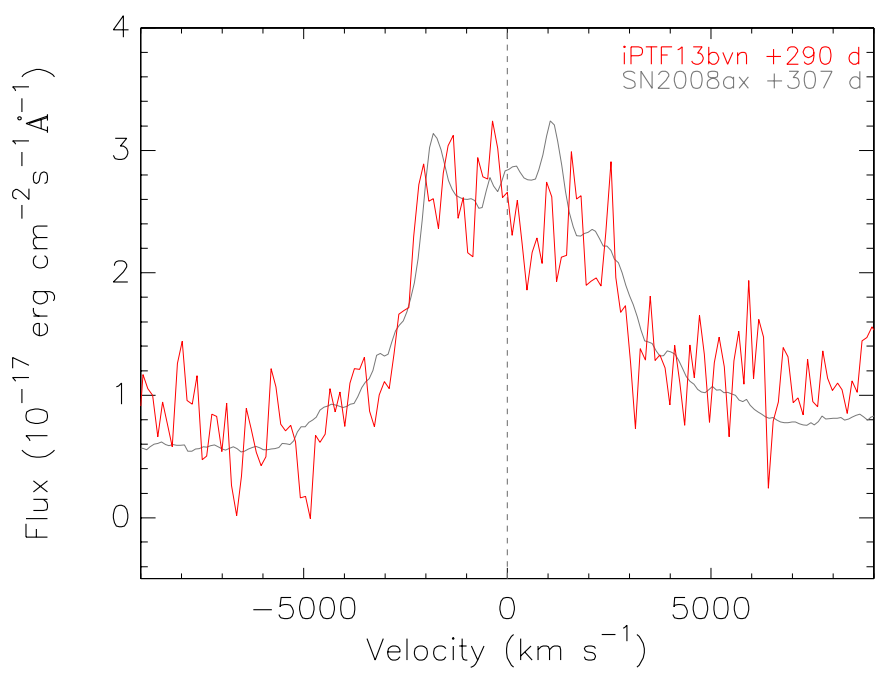

Fig. 3. Line profile of $[\mathrm{O} \mathrm{III}] \lambda \lambda 6300,6364$ of iPTF13bvn compared to SN 2008ax. The dashed vertical line corresponds to zero velocity with respect to $6300 \AA$. The flux of the spectrum of SN 2008ax has been normalized and shifted.

also shows a velocity FWHM of $\sim 5000 \mathrm{~km} \mathrm{~s}^{-1}$, in the case of SN 2008ax, the FWHM is lower, $\sim 4000 \mathrm{~km} \mathrm{~s}^{-1}$. These are within the likely core velocity values of $\sim 1000-5000 \mathrm{~km} \mathrm{~s}^{-1}$ as suggested by Fransson \& Chevalier (1989).

In Fig. 4 we present the profiles of the [O I], [Ca II], and $\mathrm{Mg}$ I] emission lines. Here we plot the observed line profile of Mg I] and additionally a synthetic line profile. This synthetic profile was generated to simulate a doublet feature for $\mathrm{Mg} \mathrm{I}$, thereby allowing direct comparison with the [O I] doublet. We follow the method described in Taubenberger et al. (2009), in which the $\mathrm{Mg} \mathrm{I}$ ] line is scaled down to one-third of its initial intensity, then shifted by $+46 \AA$ and added to the original spectrum to generate the synthetic doublet. Taubenberger et al. (2009) found that for most objects in their sample $(\sim 65 \%)$ the [O I] and $\mathrm{Mg}$ I] lines show remarkably similar profiles. It is interesting that even with a synthetic doublet, the red wing of the $\mathrm{Mg} \mathrm{I}]$ line in iPTF13bvn seems to be depressed in comparison with the profiles of [O I] and [Ca II], although there is the caveat of the low signal-to-noise ratio. The strength of this line is also considerably stronger than some other SNe as apparent in Fig. 2. The $\mathrm{Mg} \mathrm{I}] /[\mathrm{O} \mathrm{I}]$ flux ratio in iPTF13bvn is around 0.85 , whereas for SNe 2002ap, 2008ax, and 2007Y, the value is around 0.2-0.3. Foley et al. (2003) noticed the exceptional strength of this $\mathrm{Mg} \mathrm{I}]$ line in SN 2002ap at late times and its growth with time compared to the strength of [O I] and [Ca II] lines. As time passes we are seeing deeper into the progenitor core and reaching the $\mathrm{Mg}-\mathrm{O}$ layer, so it is expected that this line grows stronger at later times. For the [Ca II] line in iPTF13bvn, the line profile appears to be less asymmetric than the other two lines. These might indicate different geometries and distributions of the emitting materials for $[\mathrm{O} \mathrm{I}],[\mathrm{Ca} \mathrm{II}]$, and $[\mathrm{Mg} \mathrm{I}]$.

The profile shape of the [O I] line in iPTF13bvn suggests that there is some degree of asphericity in the ejecta. While it is not very clearly double-peaked, the line profile is considerably different to the more Gaussian-like [Ca II] line. Maeda et al. (2008) show that double-peaked [O I] $\lambda \lambda 6300,6364$ emission commonly observed in late-time spectra of core-collapse $\mathrm{SNe}$ signifies asphericity in the SN explosion (see also Modjaz et al. 2008). Tanaka et al. (2009) also report that the [O I] line in the nebular spectrum of the Type-Ib SN 2008D can be explained

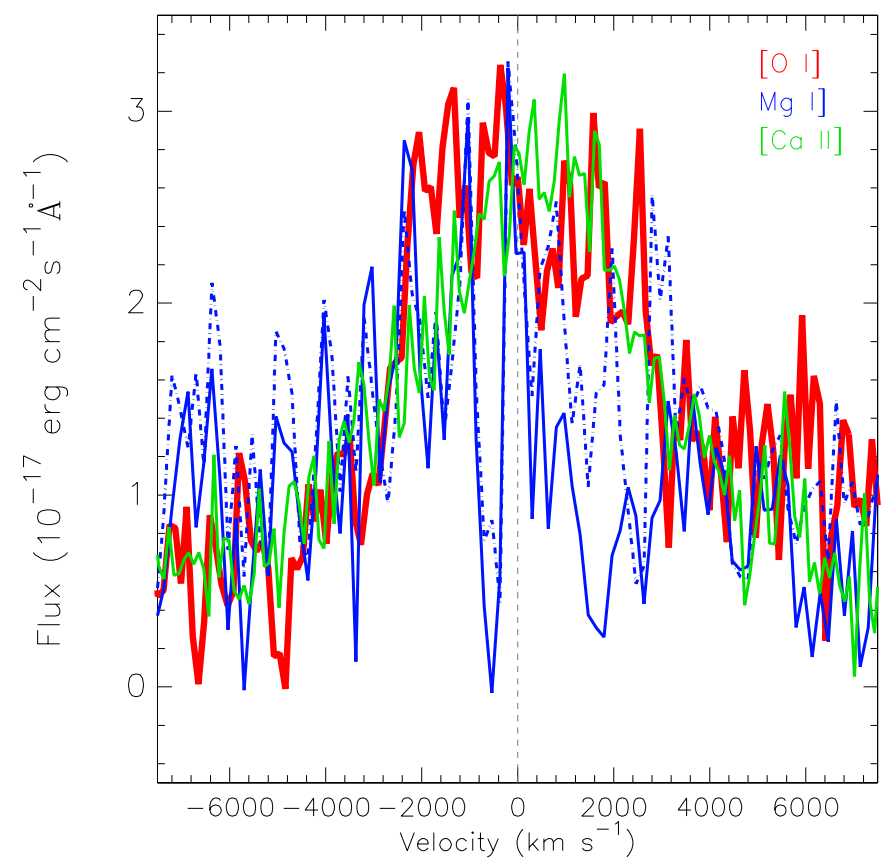

Fig. 4. Line profiles of $[\mathrm{O} \mathrm{I}],[\mathrm{Ca} I \mathrm{I}]$, and $\mathrm{Mg} \mathrm{I}]$ in velocity space. Zero velocities are with respect to $6300 \AA, 7308 \AA$, and $4571 \AA$, respectively. The fluxes of [Ca II] and $\mathrm{Mg}$ I] lines are normalized to the [O I] line. The synthetic $\mathrm{Mg}$ I] line profile is represented with dash-dotted line (see text for details).

by a bipolar explosion with a torus-like distribution of oxygen. Taubenberger et al. (2011), who also observed a similar feature in SN 2008ax, argue that a spherical oxygen distribution with a significant clump or torus, or even an aspherical distribution of ${ }^{56} \mathrm{Ni}$ that excites the oxygen, may explain the observed feature. On the other hand, the asymmetry in the line profile could be attributed to absorption in the interior, which may be caused by dust or by high opacity associated with clumping or high density material (see e.g. Taubenberger et al. 2009; Milisavljevic et al. 2010). In this case, the observed double-peaked profile may also not necessarily reflect a torus or elongated-shell geometry of the oxygen emitting region, but simply the doublet nature of the line, as suggested by Milisavljevic et al. (2010). Although it cannot be ruled out in all cases, the doublet explanation can account for neither the observed ratios of the components nor for the cases where the profile is singly peaked.

\subsubsection{Oxygen and progenitor mass}

We use the flux of the [O I] $\lambda \lambda 6300,6364$ emission line to estimate the mass of oxygen that produced the line. Jerkstrand et al. (2012) have demonstrated that the strength of the [O I] $\lambda \lambda 6300$, 6364 line is sensitive to the variation in the initial mass of the SN progenitor star.

Uomoto (1986) provided an equation to calculate the minimum oxygen mass (in $M_{\odot}$ unit) required to produce the emission, as follows:

$M_{\text {oxygen }}=10^{8} f([\mathrm{O} \mathrm{I}]) D^{2} \exp \left(2.28 / T_{4}\right)$.

Therefore, using this equation one can calculate the oxygen mass from the measured flux of the [O I] $\lambda \lambda 6300,6364$ line in erg $\mathrm{s}^{-1} \mathrm{~cm}^{-2}, f([\mathrm{O} \mathrm{I}])$, assuming that the distance in $\mathrm{Mpc}$ $D$ and temperature of the oxygen-emitting region $T_{4}$, in $10^{4} \mathrm{~K}$ 
units, are known. The distance to the host galaxy of iPTF13bvn NGC 5806 derived with Tully-Fisher method is known to be $\sim 26 \mathrm{Mpc}$. The Extragalactic Distance Database ${ }^{3}$ (Tully et al. 2009) gives the distance to NGC 5806 as $26.2 \pm 1.5 \mathrm{Mpc}$, and this has been revised with a newer value of $26.79 \pm 0.2 \mathrm{Mpc}$ in the Cosmicflows-2 catalog (Tully et al. 2013). We adopt this newer value as the distance to iPTF13bvn. For reference, Cao et al. (2013) and Srivastav et al. (2014) adopt the distance of $22.5 \mathrm{Mpc}$, while Bersten et al. (2014) uses 25.5 Mpc. From Eq. (1) it is clear that adopting a shorter distance would decrease the estimate of the oxygen mass, hence the progenitor mass.

The emission line flux of [O I] $\lambda \lambda 6300,6364$ was measured using the task splot in IRAF with three different methods. Prior to the flux measurements, we corrected the spectrum of iPTF13bvn for the Milky Way and host galaxy extinctions, amounting to $E(B-V)_{\mathrm{MW}}=0.0447 \mathrm{mag}$ and $E(B-V)_{\text {host }}=$ 0.17 mag (Bersten et al. 2014), assuming a standard Cardelli et al. (1989) interstellar reddening law with $R_{V}=3.1$, and removed the spectral continuum by fitting a first-order cubic spline function. Measurement of the line flux by direct integration gives $3.46 \times 10^{-15} \mathrm{erg} \mathrm{s}^{-1} \mathrm{~cm}^{-2}$, by fitting a single Gaussian function $3.96 \times 10^{-15} \mathrm{erg} \mathrm{s}^{-1} \mathrm{~cm}^{-2}$ and by deblending the profile into two Gaussians $3.70 \times 10^{-15} \mathrm{erg} \mathrm{s}^{-1} \mathrm{~cm}^{-2}$. We used the average of the three measurements, $3.71 \pm 0.25 \times 10^{-15} \mathrm{erg} \mathrm{s}^{-1} \mathrm{~cm}^{-2}$, as the flux of the oxygen line, to be used in Eq. (1). A possibly significant source of uncertainty in this method of Uomoto (1986) is the time evolution of the strength of the [O I] line, as well as the temperature $T_{4}$.

One way to estimate $T_{4}$ is by measuring the ratio of [O I] $\lambda 5577$ line to [O I] $\lambda \lambda 6300,6364$. However, in the case of iPTF13bvn, we could not detect this weak line at $5577 \AA$, which is actually an indication of low temperature (Elmhamdi 2011). The near-infrared Ca II line was also not detected in the iPTF13bvn spectrum, further indicating the low temperature. Sollerman et al. (1998) used this method of [O I] $\lambda 5577$ line to estimate the temperature to be $<5000-4400 \mathrm{~K}$, which in turn gives an oxygen mass of $0.11-0.21 M_{\odot}$ for SN Ib $1996 \mathrm{~N}$. After not detecting the [O I] $\lambda 5577$ in the nebular spectra of SN Ib 2009jf, Sahu et al. (2011) used $T_{4}=0.4$ to derive an oxygen mass of $1.34 M_{\odot}$. Elmhamdi et al. (2004) used the upper limit for the [O I] $\lambda 5577$ line to derive a oxygen mass of $0.7-1.35 M_{\odot}$ for SN Ib 1990I, for temperatures between $3200-3500 \mathrm{~K}$ at +237 days.

Assuming the lower limit of the temperature of the oxygenemitting region to be $3200 \mathrm{~K}$ as in SN 1990I, while adopting the line flux $3.71 \pm 0.25 \times 10^{-15} \mathrm{erg} \mathrm{s}^{-1} \mathrm{~cm}^{-2}$ and distance $26.79 \pm 0.2 \mathrm{Mpc}$, we calculated the minimum oxygen mass in iPTF13bvn using Eq. (1) to be $0.33 \pm 0.03 M_{\odot}$. This oxygen mass value is relatively low compared to SNe 1990I and 2009jf, but comparable to SN $1996 \mathrm{~N}$ if the oxygen temperature of iPTF13bvn is assumed to be slightly higher. With higher temperature, Eq. (1) will yield an even lower value of oxygen mass. Again here, we note the sensitivity of Eq. (1) to the temperature assumption: increasing the temperature from $3200 \mathrm{~K}$ to $5000 \mathrm{~K}$ will drop the derived oxygen mass by a factor of $\sim 13$, while in the opposite direction using very cool temperature less than $2500 \mathrm{~K}$ will increase the derived oxygen mass by one order of magnitude. At any rate, a temperature less than $\sim 3000 \mathrm{~K}$ is not plausible because beyond this value, the cooling will shift so to be dominated by far-infrared fine-structure lines rather than optical lines, resulting in a thermal instability and sudden temperature drop (Fransson \& Chevalier 1989). Thus, it is not likely

\footnotetext{
3 http://edd.ifa.hawaii.edu/
}

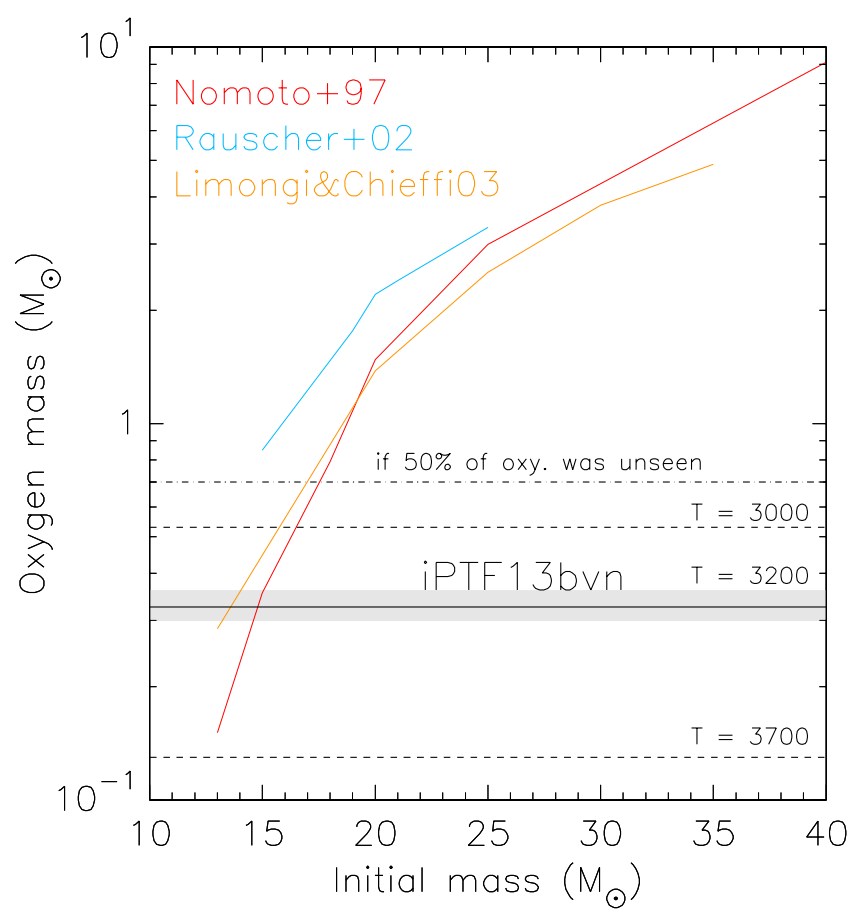

Fig. 5. Oxygen yield of the explosion of massive stars of various main sequence masses (Nomoto et al. 1997: red; Rauscher et al. 2002: light blue; Limongi \& Chieffi 2003: orange). The horizontal line indicates the estimated oxygen mass for iPTF13bvn assuming $T=3200 \mathrm{~K}$, with uncertainty represented by the shaded region. Dashed horizontal lines denote the oxygen mass values for different temperature assumptions. The estimated oxygen mass if $50 \%$ of oxygen was unseen is indicated with a dash-dotted line.

that the minimum oxygen mass is significantly higher than the derived value of $0.33 M_{\odot}$.

It is to be noted, however, that Eq. (1) provides the minimum oxygen mass responsible for the line emission, which may not be equal to the total oxygen mass. The presence of non-optically thin materials and clumping is not accounted for, thus the total oxygen mass may actually be higher than the derived value of $0.33 M_{\odot}$. Maeda et al. (2007a) derived a total oxygen mass of $2.3 M_{\odot}$ for SN 2006aj, 1.3 $M_{\odot}$ of which is within $v \lesssim 7300 \mathrm{~km} \mathrm{~s}^{-1}$ and consistent with the luminosity of the [O I] $\lambda \lambda 6300,6364$ lines. With a conservative assumption that $\sim 50 \%$ of the total oxygen mass in iPTF13bvn is responsible for the emission line, the total oxygen mass should then not exceed $\sim 0.7 M_{\odot}$. The effects of temperature assumption and nonemissive oxygen seem to dominate the uncertainty in the oxygen mass derivation, compared with the uncertainties in distance or flux measurement.

In Fig. 5 we compare the derived oxygen mass with nucleosynthesis oxygen yields of massive stars of various initial masses from Nomoto et al. (1997), Rauscher et al. (2002), and Limongi \& Chieffi (2003). It is apparent that the derived oxygen mass of iPTF13bvn is more consistent with what would be produced from the explosion of a massive star with mass $\lesssim 15-17 M_{\odot}$, even if the total oxygen mass is assumed to be twice the derived value from the $[\mathrm{O}$ I $] \lambda \lambda 6300,6364$ line. In an extreme case in which the whole SN ejecta $\left(\$ 2.3 M_{\odot}\right.$; Bersten et al. 2014; Srivastav et al. 2014) were assumed to be oxygen, which is not realistic, the initial progenitor mass of iPTF13bvn would still be lower than $\sim 25 M_{\odot}$. For reference, the derived oxygen masses of SNe 1990I and 2009jf are around 14\% to 36\% of the total ejecta mass (Elmhamdi et al. 2004; Sahu et al. 2011). 


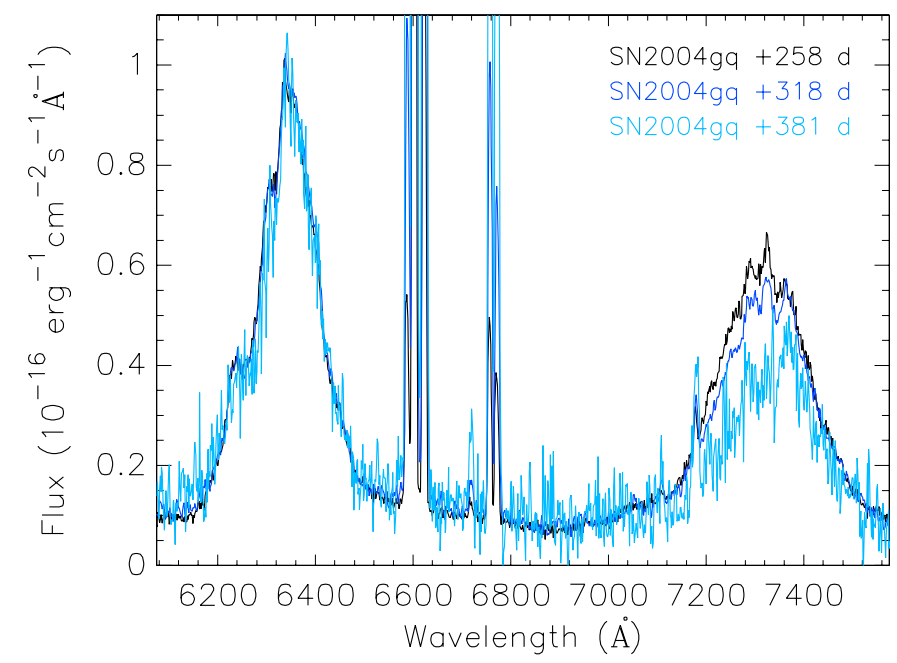

Fig. 6. Evolution of the nebular spectrum of SN 2004gq around the $[\mathrm{O} \mathrm{I}]-[\mathrm{Ca}$ II] spectral region, between +258 and +381 days post maximum. Spectra have been scaled to match the [O I ] line at +258 days. Data from Maeda et al. (2008).

This implies that a single Wolf-Rayet progenitor scenario for iPTF13bvn, whose initial mass must have been $\gtrsim 25 M_{\odot}$ at solar metallicity (Crowther 2007), is unlikely. Indeed, a lower mass binary progenitor scenario for iPTF13bvn seems to be more plausible (Bersten et al. 2014; Fremling et al. 2014; Srivastav et al. 2014; Eldridge et al. 2015). With lower metallicity, the WR mass limit would move to even higher mass owing to the reduced strength of the metallicity-driven wind. In this context, the metallicity of the explosion site of iPTF13bvn was measured to be slightly less than solar (Sect. 3.1.4). We note that nucleosynthesis yields are possibly subject to the uncertainties introduced by neglecting the effects of turbulence in the interior (Smith \& Arnett 2014), so this may affect the estimate of the initial mass of the SN progenitor star. However, this effect still has not been thoroughly studied and quantified.

We also measured the line ratio of [O I] $\lambda \lambda 6300,6364 /$ [Ca II] $\lambda \lambda 7291,7324$ emissions in the nebular spectrum of iPTF13bvn and compared it to other stripped-envelope $\mathrm{SNe}$, as well as several Type-II SNe taken from the SUSPECT ${ }^{4}$ Online Supernova Spectrum Archive. This line ratio is known to be insensitive to the density and temperature, while increases with increasing progenitor mass (Fransson \& Chevalier 1989; also see Elmhamdi et al. 2004). The evolution of the ratio of this line with SN age is generally low, as shown in an example of SN 2004gq in Fig. 6. The ratio of [O I $] \lambda \lambda 6300,6364 /[\mathrm{Ca}$ II $] \lambda \lambda 7291$, 7324 changes from around unity at phase +258 days to 1.3 at +381 days; i.e., only $\sim 30 \%$ change in more than 120 days. It has also been shown by Elmhamdi et al. (2004, see their Fig. 3) that the ratio is almost constant for $\mathrm{SNe}$ aged 280-400 days after the explosion. Figure 7 shows the measured line ratio for iPTF13bvn compared to other SNe. It is clear that the SNe II do not exceed line ratio of 0.7 , while on the other hand, there is considerable spread for the Type-Ib/Ic SNe. While the evolution of the line ratio may contribute to the scatter in the diagram, it is considerably insignificant compared to the observed spread ${ }^{5}$.

\footnotetext{
4 http://www.nhn.ou.edu/ suspect/

5 We, however, again caution the reader of the caveat that it is possible that this pattern observed in Fig. 7 may change with time as different $\mathrm{SNe}$ may exhibit different rates of evolution, i.e. some objects may evolve more dramatically compared to the others. Note that this late-time behaviour in SNe is still not very well observed and studied.
}

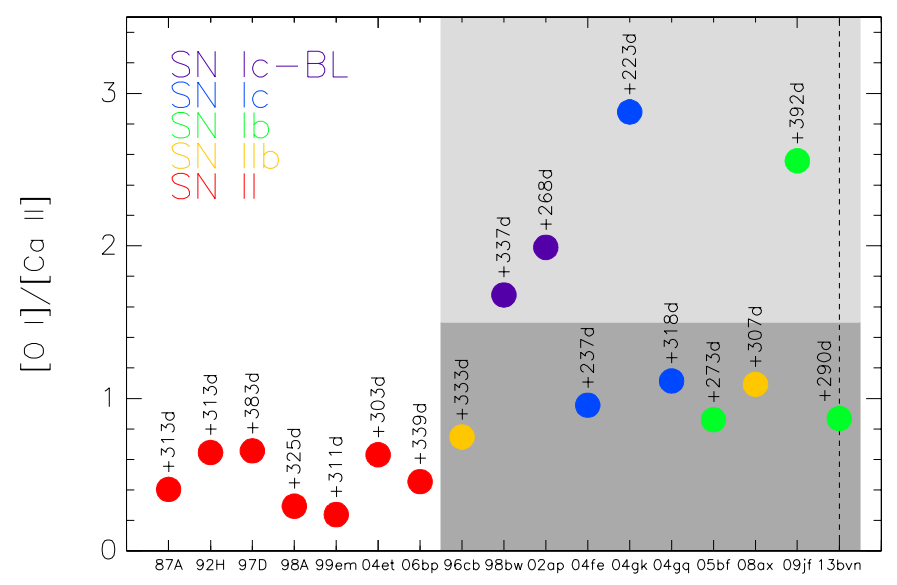

Fig. 7. [O I] $] \lambda \lambda 6300,6364 /[\mathrm{Ca}$ II] $] \lambda \lambda 7291,7324$ line ratio of several corecollapse $\mathrm{SNe}$ of similar ages during the nebular phase. Red symbols indicate SN Type-II, orange Type-IIb, green Type-Ib, blue Type-Ic, and purple broad-lined Type-Ic. The SN phases with respect to the time of maximum light are shown next to the each data point and the dashed vertical line indicates iPTF13bvn. The light-grey shaded region indicates the single star progenitors and the dark-grey shaded region indicates the binary progenitors for Type-Ib/c SNe. The exact border between these two regions is unknown, thus arbitrarily taken at $[\mathrm{O} \mathrm{I}] /[\mathrm{Ca} \mathrm{II}]=1.5$ in this plot for the purpose of indicating the two distinct populations. References for the spectra of individual SNe: 1987A (Pun et al. 1995); 1992H (Clocchiatti et al. 1996); 1997D (Benetti et al. 2001); 1998A (Pastorello et al. 2005); 1998bw (Patat et al. 2001); $1999 \mathrm{em}$ (Leonard et al. 2002); 2004et (Sahu et al. 2006); 2004gq (Maeda et al. 2008); 2005bf (Maeda et al. 2007b); 2006bp (Quimby et al. 2007); 1996cb, 2002ap, 2004fe, 2004gk, 2008ax, 2009jf (Modjaz et al. 2008, 2014).

This spread may be interpreted as indicating the presence of two different populations of $\mathrm{SNe} \mathrm{Ib} / \mathrm{c}$ (shaded regions in Fig. 7), i.e. those coming from massive single Wolf-Rayet progenitors, and lower-mass progenitors in binary systems (Kuncarayakti et al. 2013). In this case, iPTF13bvn appears to be more associated to the lower mass progenitors of Type-II SNe, further strengthening the notion of its progenitor in a binary system. It has been suggested that the majority ( $\gtrsim 70 \%$ ) of massive stars undergo binary interaction at some point in their evolutions, thus implying that a significant fraction of core-collapse SN progenitors must experience such phases as well (Sana et al. 2012). The confirmation that the progenitor of iPTF13bvn indeed belonged to a binary system may eventually be provided by the detection of the remaining companion by future observations when the SN has faded considerably, such as the case of SN IIb 2011dh (Folatelli et al. 2014b).

\subsubsection{Possible presence of $\mathrm{H} \alpha$ ?}

As can be seen in Fig. 2, the overall appearance of the late-time spectrum of iPTF13bvn closely resembles that of SN 2008ax. Taubenberger et al. (2011) report the presence of a broad feature redwards of the [O I] $\lambda \lambda 6300,6364$ emission line in the nebular spectra of SN 2008ax. This feature appeared $~ 100$ days after the explosion and remained visible up to at least phase 358 days. In other stripped-envelope $\mathrm{SNe}$, this feature has also been observed, such as in SN IIb 1993J (Patat et al. 1995) and SN Ib 1996N (Sollerman et al. 1998). In the nebular spectra of SN Ib 2005bf (Maeda et al. 2007b), a broad feature at $\sim 6500 \AA$ has been identified as $\mathrm{H} \alpha$ (see Fig. 2). Also in the case of SN 2010as, a lowvelocity SN IIb showing Ib/c characteristics, this feature was 
H. Kuncarayakti et al.: Nebular phase observations of the Type-Ib supernova iPTF13bvn favour a binary progenitor

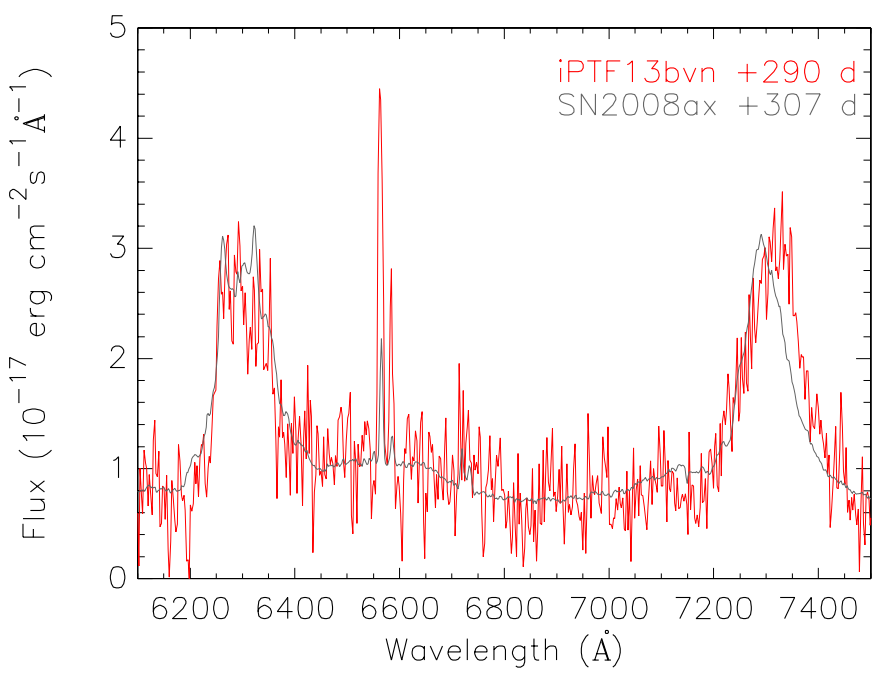

Fig. 8. Spectra of iPTF13bvn and SN 2008ax around the $\mathrm{H} \alpha$ region. The flux of the SN 2008ax spectrum has been normalized and shifted.

also observed (Folatelli et al. 2014a). During the transitional phase from photospheric to nebular at around +100 days past maximum light, the profile is complex, possibly indicating the presence of other lines, and it appears to be still present well into the nebular phase at +309 days.

Although it is compelling to attribute this feature to latetime $\mathrm{H} \alpha$ emission from the $\mathrm{SN}$, as discussed by Taubenberger et al. (2011), there could be other possible sources of this broad feature, such as various other elements including Fe and scattering. In Fig. 8 we plot the spectrum of iPTF13bvn alongside SN 2008ax from Modjaz et al. (2014, same data as shown in Fig. 2) around the $\mathrm{H} \alpha$ region. The graph is suggestive of a broad feature redwards of the [O I] line in the iPTF13bvn spectrum, very similar to SN 2008ax. However, owing to the possible ambiguity in the identification as discussed above and low signal-to-noise ratio in the spectrum, we restrained from firmly associating this feature with $\mathrm{H} \alpha$ emission from the $\mathrm{SN}$ itself. Furthermore, Jerkstrand et al. (2015) argue that the thin hydrogen envelope in Type-IIb SNe should not affect the spectrum after around 150 days. They attribute the feature redwards of the [O I] line in the nebular spectra of SNe 1993J, 2008ax, and $2001 \mathrm{dh}$ to emission from [N II] $\lambda \lambda 6548,6583$.

We note that the early-phase spectra of iPTF13bvn bear similarities to SNe 2007Y and 2010as, which were shown to belong to the class of "flat-velocity Type-IIb" transitional SNe (Folatelli et al. 2014a). These objects show SN Type-Ib/c signatures in their spectra, but also the presence of hydrogen and a characteristic flat velocity evolution between 6000 and $8000 \mathrm{~km} \mathrm{~s}^{-1}$. The velocity evolution of iPTF13bvn, however, does not show this characteristic flat evolution (see Fig. 9 of Srivastav et al. 2014). In Fig. 9 we show the early spectra of 13bvn, around one week before and after maximum light compared to other SNe including 2007Y and 2010as. The absorption trough at $6200 \AA$ in iPTF13bvn has been attributed to possibly $\mathrm{Si}$ II or $\mathrm{Ne}$ I by Srivastav et al. (2014) and Cao et al. (2013). In the case of SN 2010as, it was shown that this absorption is best matched with high-velocity $\mathrm{H} \alpha$ rather than with $\mathrm{Si}$ II (Folatelli et al. 2014a).

\subsubsection{H II region metallicity}

There are narrow emission lines in the spectrum of iPTF13bvn. The emission lines of $\mathrm{H} \alpha$, [N II] $\lambda 6583$, and [S II] $\lambda \lambda 6716,6731$ are presumably produced by the underlying $\mathrm{H}$ II region at the $\mathrm{SN}$ position. Measurement of the logarithm of the flux ratio of [N II] to $\mathrm{H} \alpha$ emission lines, the $N 2$ index, can be used as an indicator of metallicity. Pettini \& Pagel (2004) gives the oxygen abundance as $12+\log (\mathrm{O} / \mathrm{H})=8.90+0.57 \times N 2$. Our measurement of the $N 2$ index shows that the $\mathrm{H}$ II region associated with iPTF13bvn has the gas-phase metallicity of $12+\log (\mathrm{O} / \mathrm{H})=8.63$. This suggests that the metallicity of the progenitor of iPTF13bvn is close to the solar value of $12+\log (\mathrm{O} / \mathrm{H})=8.69$ (Asplund et al. 2009), i.e. about $0.87 Z_{\odot}$.

We also measured the oxygen abundance from a nearby $\mathrm{H}$ II region 0.7 arcsec away from the $\mathrm{SN}$, corresponding to a projected physical distance of $89 \mathrm{pc}$. We used the data taken as part of the Carnegie Supernova Project (CSP; Hamuy et al. 2006), using the $6.5 \mathrm{~m}$ Magellan Baade telescope at Las Campanas Observatory, Chile, and the instrument IMACS (Bigelow et al. 1998) on longslit spectroscopy mode with 300 lines/mm grating blazed at $4.3^{\circ}$. The observation was carried out in the night of 13 August 2014 (local time), under 0.5 arcsec seeing. Measurement of the $N 2$ index yields $12+\log (\mathrm{O} / \mathrm{H})=8.62$, which is very close to the derived on-site metallicity of 8.63 .

As already mentioned in Sect. 3.1.2, at solar metallicity the minimum initial mass for a single star to become a Wolf-Rayet star via stellar wind is $\sim 25 M_{\odot}$ (Crowther 2007). Considering the metallicity estimate and core oxygen mass, it is unlikely that iPTF13bvn was produced by a $\gtrsim 25 M_{\odot}$ progenitor.

\subsection{Late-time light curve}

Our photometry unfortunately sampled only one epoch during the late-time evolution of iPTF13bvn. In Fig. 10 we plot our $U B V R$ c data points at 306 days, together with the light curve constructed using the published photometric data of Srivastav et al. (2014). Assuming a constant decay rate since the latest points in the early light curves $(+87$ days in $R$ and $V,+52$ days in $B$ ), the late-time tails of the light curves appear to decay more rapidly than the ${ }^{56} \mathrm{Co}$ decay rate of $0.98 \mathrm{mag}(100 \mathrm{~d})^{-1}$ (Woosley et al. 1989), i.e. $1.32 \mathrm{mag}, 1.55 \mathrm{mag}$, and $1.13 \mathrm{mag}(100 \mathrm{~d})^{-1}$ in the $R, V$, and $B$ bands, respectively.

The steeper light curve decay compared to ${ }^{56} \mathrm{Co}$ signifies that complete $\gamma$-ray trapping did not occur in the case of iPTF13bvn. This is a common phenomenon observed in other strippedenvelope SNe during the nebular phase - for example SN 2008ax (Taubenberger et al. 2011), SN 1996N (Sollerman et al. 1998), and SN 1990I (Elmhamdi et al. 2004) - and is associated with relatively low ejecta mass. The decline of the light curve of SN 1990I in the nebular phase is the steepest in the $V$ band, followed by $R$ and then $B$, which is very likely also the case in iPTF13bvn, although the lack of data points during the late-time evolution of the light curve prevents us from drawing this conclusion.

\section{Summary}

We present late-time photometric and spectroscopic observations of the Type-Ib SN iPTF13bvn. A progenitor candidate of this $\mathrm{SN}$ has been detected in pre-explosion HST images, which is potentially the first for Type Ib/c SNe. Our spectroscopy shows that the nebular spectrum of the SN is dominated by emission lines of $\mathrm{Mg} \mathrm{I}$, [O I], and [Ca II]. Measurement of the flux of the [O I] $\lambda \lambda 6300,6464$ line shows that the oxygen mass of iPTF13bvn does not significantly exceed $\sim 0.7 M_{\odot}$, assuming 

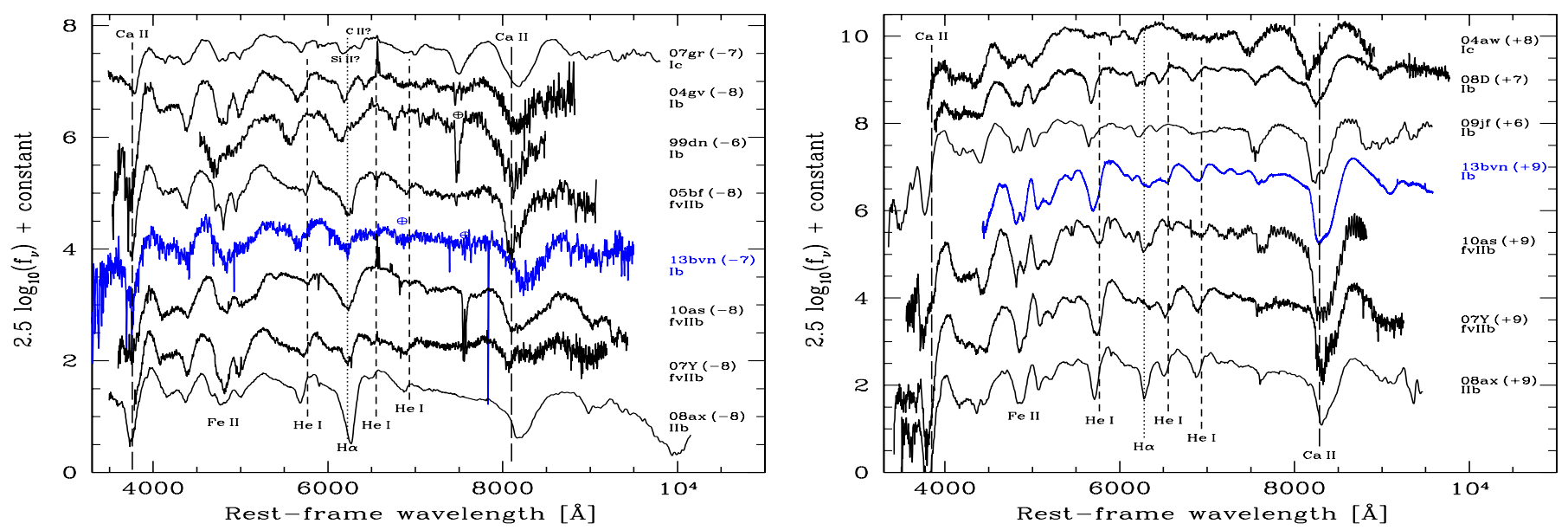

Fig. 9. Early spectra of iPTF13bvn at around one week before and after maximum light, compared to other stripped-envelope SNe. Vertical dashed lines show the positions of prominent helium lines, and the dotted line shows the position of high-velocity H $\alpha$. The spectra were taken from the Weizmann Interactive Supernova Data Repository (WISeREP; Yaron \& Gal-Yam 2012)

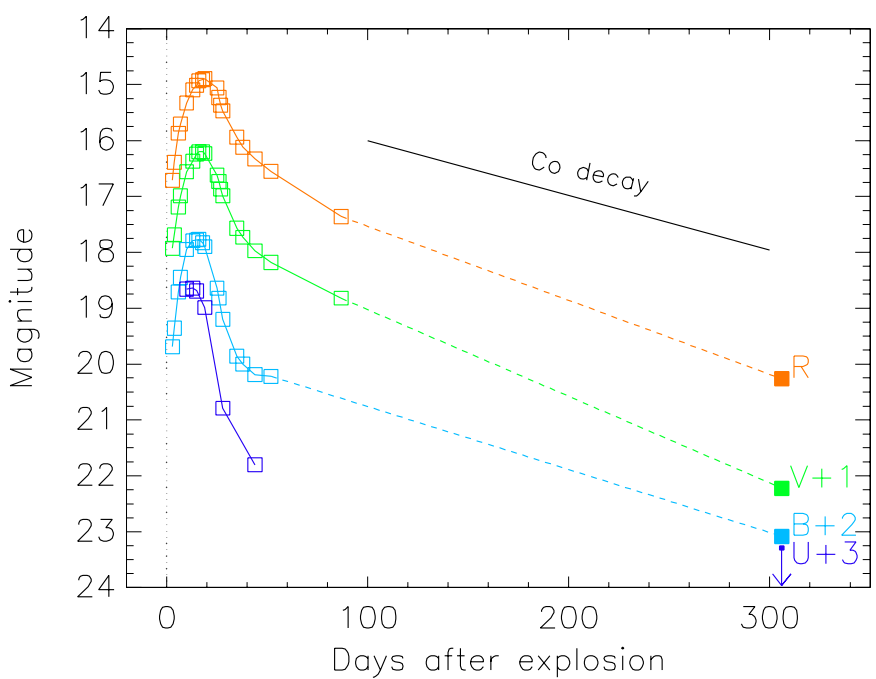

Fig. 10. Light curve of iPTF13bvn up to Day 306 after the explosion. Explosion time is indicated with a dotted vertical line. Early photometric data from Srivastav et al. (2014) are plotted in open squares, and the latest points in the early light curves are connected to our data points (filled squares) in the nebular phase by dashed lines. Typical photometric error bars are smaller than the plotting symbol size.

that the emission is produced by $50 \%$ of the total oxygen mass, suggesting that the progenitor star could not have been more massive than $\sim 15-17 M_{\odot}$. The uncertainty stemmed from distance estimate, and oxygen line flux measurements are negligible compared to the error from temperature and unseen oxygen. Comparing the nebular [O I]/[Ca II] line ratio with other SNe, iPTF13bvn appears to be associated with low-mass progenitor stars.

This piece of evidence provides another support to the scenario in which iPTF13bvn was produced by a hydrogen-poor progenitor star in a binary system that has undergone envelope stripping by means of close binary interaction. The initial mass of the progenitor star was not high enough for the star to have become a Wolf-Rayet star, if it were a single star. This result shows very good agreement with the results from other methods in constraining the progenitor star, such as from hydrodynamical modelling (Bersten et al. 2014), analytical modelling
(Srivastav et al. 2014), and binary evolution modelling (Bersten et al. 2014; Eldridge et al. 2015). In the context of hydrogen-poor $\mathrm{SNe}$, iPTF13bvn further reinforces the importance of massive close binaries as a prominent, if not the dominant, progenitors of these SNe.

Acknowledgements. We thank the anonymous referee for useful comments that substantially improved this paper. Support for H.K., S.G.G., M.H., T.d.J., and C.P.G. is provided by the Ministry of Economy, Development, and Tourism's Millennium Science Initiative through grant IC120009, awarded to The Millennium Institute of Astrophysics, MAS. H.K. and S.G.G. acknowledge support by CONICYT through FONDECYT grants 3140563 and 3130680, respectively. H.K. also acknowledges helpful support from the staffs of Cerro Pachón and NOAO South during the observing run. K.M. acknowledges financial support by Grant-in-Aid for Scientific Research (No. 23740141 and 26800100) from the Japanese Ministry of Education, Culture, Sports, Science and Technology (MEXT). The work by K.M. is partly supported by WPI Initiative, MEXT, Japan. E.Y.H. acknowledges the generous support provided by the Danish Agency for Science and Technology and Innovation through a Sapere Aude Level 2 grant. This research made use of the NASA/IPAC Extragalactic Database (NED), which is operated by the Jet Propulsion Laboratory, California Institute of Technology, under contract with the National Aeronautics and Space Administration.

\section{References}

Asplund, M., Grevesse, N., Sauval, A. J., \& Scott, P. 2009, ARA\&A, 47, 481 Benetti, S., Turatto, M., Balberg, S., et al. 2001, MNRAS, 322, 361 Bersten, M. C., Benvenuto, O. G., Folatelli, G., et al. 2014, AJ, 148, 68 Bigelow, B. C., Dressler, A. M., Shectman, S. A., \& Epps, H. W. 1998, Proc. SPIE, 3355, 225

Cao, Y., Kasliwal, M. M., Arcavi, I., et al. 2013, ApJ, 775, L7

Cardelli, J. A., Clayton, G. C., \& Mathis, J. S. 1989, ApJ, 345, 245

Clemens, J. C., Crain, J. A., \& Anderson, R. 2004, Proc. SPIE, 5492, 331

Clocchiatti, A., Benetti, S., Wheeler, J. C., et al. 1996, AJ, 111, 1286

Crowther, P. A. 2007, ARA\&A, 45, 177

Eldridge, J. J., Fraser, M., Smartt, S. J., Maund, J. R., \& Crockett, R. M. 2013, MNRAS, 436, 774

Eldridge, J. J., Fraser, M., Maund, J. R., \& Smartt, S. J. 2015, MNRAS, 446, 2689

Elmhamdi, A. 2011, Acta Astron., 61, 179

Elmhamdi, A., Danziger, I. J., Cappellaro, E., et al. 2004, A\&A, 426, 963

Ensman, L. M., \& Woosley, S. E. 1988, ApJ, 333, 754

Folatelli, G., Bersten, M. C., Kuncarayakti, H., et al. 2014a, ApJ, 792, 7

Folatelli, G., Bersten, M. C., Benvenuto, O. G., et al. 2014b, ApJ, 793, L22

Foley, R. J., Papenkova, M. S., Swift, B. J., et al. 2003, PASP, 115, 1220

Fransson, C., \& Chevalier, R. A. 1989, ApJ, 343, 323

Fremling, C., Sollerman, J., Taddia, F., et al. 2014, A\&A, 565, A114

Groh, J. H., Georgy, C., \& Ekström, S. 2013, A\&A, 558, L1

Hamuy, M., Folatelli, G., Morrell, N. I., et al. 2006, PASP, 118, 2

Jerkstrand, A., Fransson, C., Maguire, K., et al. 2012, A\&A, 546, A28 
H. Kuncarayakti et al.: Nebular phase observations of the Type-Ib supernova iPTF13bvn favour a binary progenitor

Jerkstrand, A., Ergon, M., Smartt, S. J., et al. 2015, A\&A, 573, A12 Kuncarayakti, H., Doi, M., Aldering, G., et al. 2013, AJ, 146, 30 Landolt, A. U. 1992, AJ, 104, 340

Law, N. M., Kulkarni, S. R., Dekany, R. G., et al. 2009, PASP, 121, 1395 Leonard, D. C., Filippenko, A. V., Gates, E. L., et al. 2002, PASP, 114, 35 Limongi, M., \& Chieffi, A. 2003, ApJ, 592, 404

Maeda, K., Kawabata, K., Tanaka, M., et al. 2007a, ApJ, 658, L5

Maeda, K., Tanaka, M., Nomoto, K., et al. 2007b, ApJ, 666, 1069

Maeda, K., Kawabata, K., Mazzali, P. A., et al. 2008, Science, 319, 1220

Maeder, A., \& Lequeux, J. 1982, A\&A, 114, 409

Milisavljevic, D., Fesen, R. A., Gerardy, C. L., Kirshner, R. P., \& Challis, P. 2010, ApJ, 709, 1343

Milisavljevic, D., Margutti, R., Soderberg, A. M., et al. 2013, ApJ, 767, 71

Modjaz, M., Kirshner, R. P., Blondin, S., Challis, P., \& Matheson, T. 2008, ApJ, 687, L9

Modjaz, M., Blondin, S., Kirshner, R. P., et al. 2014, AJ, 147, 99

Nomoto, K., Hashimoto, M., Tsujimoto, T., et al. 1997, Nucl. Phys. A, 616, 79

Pastorello, A., Baron, E., Branch, D., et al. 2005, MNRAS, 360, 950

Patat, F., Chugai, N., \& Mazzali, P. A. 1995, A\&A, 299, 715

Patat, F., Cappellaro, E., Danziger, J., et al. 2001, ApJ, 555, 900

Pettini, M., \& Pagel, B. E. J. 2004, MNRAS, 348, L59

Pun, C. S. J., Kirshner, R. P., Sonneborn, G., et al. 1995, ApJS, 99, 223
Quimby, R. M., Wheeler, J. C., Höflich, P., et al. 2007, ApJ, 666, 1093 Rau, A., Kulkarni, S. R., Law, N. M., et al. 2009, PASP, 121, 1334

Rauscher, T., Heger, A., Hoffman, R. D., \& Woosley, S. E. 2002, ApJ, 576, 323

Sahu, D. K., Anupama, G. C., Srividya, S., \& Muneer, S. 2006, MNRAS, 372 1315

Sahu, D. K., Gurugubelli, U. K., Anupama, G. C., \& Nomoto, K. 2011, MNRAS, 413,2583

Sana, H., de Mink, S. E., de Koter, A., et al. 2012, Science, 337, 444

Smith, N., \& Arnett, W. D. 2014, ApJ, 785, 82

Sollerman, J., Leibundgut, B., \& Spyromilio, J. 1998, A\&A, 337, 207

Srivastav, S., Anupama, G. C., \& Sahu, D. K. 2014, MNRAS, 445, 1932

Stetson, P. B. 1987, PASP, 99, 191

Tanaka, M., Yamanaka, M., Maeda, K., et al. 2009, ApJ, 700, 1680

Taubenberger, S., Valenti, S., Benetti, S., et al. 2009, MNRAS, 397, 677

Taubenberger, S., Navasardyan, H., Maurer, J. I., et al. 2011, MNRAS, 413, 2140

Tully, R. B., Rizzi, L., Shaya, E. J., et al. 2009, AJ, 138, 323

Tully, R. B., Courtois, H. M., Dolphin, A. E., et al. 2013, AJ, 146, 86

Uomoto, A. 1986, ApJ, 310, L35

Woosley, S. E., Hartmann, D., \& Pinto, P. A. 1989, ApJ, 346, 395

Yaron, O., \& Gal-Yam, A. 2012, PASP, 124, 668

Yoon, S.-C., Gräfener, G., Vink, J. S., Kozyreva, A., \& Izzard, R. G. 2012, A\&A, 544, L11 